Georgian Mathematical Journal

Volume 8 (2001), Number 4, 753-766

\title{
UNILATERAL CONTACT OF ELASTIC BODIES (MOMENT THEORY)
}

\author{
R. GACHECHILADZE
}

\begin{abstract}
Boundary contact problems of statics of the moment (couplestress) theory of elasticity are studied in the case of a unilateral contact of two elastic anisotropic nonhomogeneous media. A problem, in which during deformation the contact zone lies within the boundaries of some domain, and a problem, in which the contact zone can extend, are given a separate treatment. Concrete problems suitable for numerical realizations are considered.
\end{abstract}

2000 Mathematics Subject Classification: 74B99, 35J85.

Key words and phrases: Couple-stress elasticity, boundary contact problems, statics, one-sided contact, variational inequality.

The theory of variational inequalities is one of the most successful methods used to investigate problems of the unilateral contact of elastic bodies. In this paper, using the general theory of variational inequalities, we study the unilateral contact of two anisotropic nonhomogeneous elastic bodies in the moment theory of elasticity. We also study questions of the existence and uniqueness of a weak solution and consider concrete problems suitable for numerical realizations. A problem in which the contact zone lies within some domain and a problem in which the contact zone extends are treated separately.

\section{A Problem with a Bounded Contact Zone}

Let $\Omega_{q} \subset \mathbb{R}^{3}(q=1,2)$ be the contacting bounded domains with Lipshitz piecewise smooth boundaries filled up with different elastic materials. The basic equations of statics of the couple-stress theory of elasticity for anisotropic nonhomogeneous elastic media theory can be written in terms of stress components as follows (see [1]):

$$
\begin{aligned}
& \partial \sigma_{i j}^{(q)}(x) / \partial x_{i}+F_{j}^{(q)}(x)=0, \\
& \partial \mu_{i j}^{(q)}(x) / \partial x_{i}+\varepsilon_{i k j} \sigma_{i k}^{(q)}(x)+G_{j}^{(q)}(x)=0, \quad x \in \Omega_{q}, \quad q=1,2,
\end{aligned}
$$

where $\varepsilon_{i k j}$ is the Levy-Cività symbol, $F^{(q)}: \Omega_{q} \rightarrow \mathbb{R}^{3}$ is mass force, $G^{(q)}: \Omega_{q} \rightarrow$ $\mathbb{R}^{3}$ is mass moment; $\sigma_{i j}^{(q)}, \mu_{i j}^{(q)}: \Omega_{q} \rightarrow \mathbb{R}$, where

$$
\sigma_{i j}^{(q)}(x)=a_{i j l k}^{(q)}(x) u_{l k}^{(q)}(x)+b_{i j l k}^{(q)}(x) \omega_{l k}^{(q)}(x)
$$

ISSN 1072-947X / \$8.00 / C) Heldermann Verlag www.heldermann.de 
is the stress force tensor, and

$$
\mu_{i j}^{(q)}(x)=b_{l k i j}^{(q)}(x) u_{l k}^{(q)}(x)+c_{i j l k}^{(q)}(x) \omega_{l k}^{(q)}(x)
$$

is the moment stress tensor (here and in what follows the repetition of the symbol without brackets means summation over this index from 1 to 3 ),

$$
u_{l k}^{(q)}(x)=\partial u_{k}^{(q)}(x) / \partial x_{l}-\varepsilon_{l k m} \omega_{m}^{(q)}(x)
$$

are strain components, $\omega_{l k}^{(q)}(x)=\partial \omega_{k}^{(q)}(x) / \partial x_{l}$ are torsion-bending components, $u^{(q)}: \Omega_{q} \rightarrow \mathbb{R}^{3}$ is the displacement vector, $\omega^{(q)}: \Omega_{q} \rightarrow \mathbb{R}^{3}$ is the rotation vector, and the real-valued functions $a_{i j l k}^{(q)}, b_{i j l k}^{(q)}, c_{i j l k}^{(q)}$ (called the elastic constants) belong to the class $C^{1}\left(\bar{\Omega}_{q}\right)$ and satisfy the usual requirements for symmetry and ellipticity, i.e., $a_{i j l k}^{(q)}=a_{l k i j}^{(q)}, \quad c_{i j l k}^{(q)}=c_{l k i j}^{(q)}, \quad q=1,2$, and there exists $\alpha_{0}>0$ such that

$$
a_{i j l k}^{(q)}(x) \xi_{i j} \xi_{l k}+2 b_{i j l k}^{(q)}(x) \xi_{i j} \eta_{l k}+c_{i j l k}^{(q)}(x) \eta_{i j} \eta_{l k} \geq \alpha_{0}\left(\xi_{i j} \xi_{i j}+\eta_{i j} \eta_{i j}\right)
$$

for all $x \in \bar{\Omega}_{q}$ and $\xi_{i j}, \eta_{i j} \in \mathbb{R}$.

Let $n^{(q)}(x), q=1,2$ be the unit normal at a point $x$ of the surface $\partial \Omega_{q}$ which is external with respect to $\Omega^{(q)}$, and $\stackrel{(q)}{\mathcal{M}}(x, \partial)$ be the $6 \times 6$ matrix differential operator of statics of the couple-stress theory of elasticity which corresponds to system (1) (see [2], [3]), and $\stackrel{(q)}{\mathcal{N}}\left(x, \partial, n^{(q)}\right)$ be the $6 \times 6$ matrix differential stress operator (see [2], [3]). Clearly, in such notation, system (1) takes the form

$$
\stackrel{(q)}{\mathcal{M}}(x, \partial) \mathcal{U}^{(q)}(x)+\mathcal{F}^{(q)}(x)=0, \quad x \in \Omega_{q}, \quad q=1,2,
$$

where $\mathcal{U}^{(q)}=\left(u^{(q)}, \omega^{(q)}\right)$ and $\mathcal{F}^{(q)}=\left(F^{(q)}, G^{(q)}\right)$.

We will use the following notation:

$\stackrel{(q)}{\sigma}_{\mathcal{U}^{(q)}} \equiv \stackrel{(q)}{\mathcal{N}}^{1}\left(x, \partial, n^{(q)}\right) u^{(q)}(x)+\stackrel{(q)}{\mathcal{N}}^{2}\left(x, \partial, n^{(q)}\right) \omega^{(q)}(x)$ is the force stress vector;

$\stackrel{(q)}{\mu} \mathcal{U}^{(q)} \equiv \stackrel{(q)}{\mathcal{N}}^{3}\left(x, \partial, n^{(q)}\right) u^{(q)}(x)+\stackrel{(q)}{\mathcal{N}}^{4}\left(x, \partial, n^{(q)}\right) \omega^{(q)}(x)$ is the stress moment vector, where $\stackrel{(q)}{\mathcal{N}}^{j}, j=1,2,3,4$, are the $3 \times 3$ matrix differential operators participating in the definition of the operator $\stackrel{(q)}{\mathcal{N}}\left(x, \partial, n^{(q)}\right)$ (see [2], [3]).

The total strain energy of the respective media has the form

$$
\begin{aligned}
\mathcal{B}^{(q)}\left(\mathcal{U}^{(q)}, \mathcal{V}^{(q)}\right) & =\int_{\Omega_{q}}\left\{a_{i j l k}^{(q)}(x) \xi_{i j}\left(\mathcal{U}^{(q)}\right) \xi_{l k}\left(\mathcal{V}^{(q)}\right)+c_{i j l k}^{(q)}(x) \eta_{i j}\left(\mathcal{U}^{(q)}\right) \eta_{l k}\left(\mathcal{V}^{(q)}\right)\right. \\
& \left.+b_{i j l k}^{(q)}(x) \xi_{i j}\left(\mathcal{U}^{(q)}\right) \eta_{l k}\left(\mathcal{V}^{(q)}\right)+b_{i j l k}^{(q)}(x) \xi_{i j}\left(\mathcal{V}^{(q)}\right) \eta_{l k}\left(\mathcal{U}^{(q)}\right)\right\} d x,
\end{aligned}
$$

where

$$
\xi_{i j}\left(\mathcal{U}^{(q)}\right)=\frac{\partial u_{j}^{(q)}}{\partial x_{i}}-\varepsilon_{i j k} \omega_{k}^{(q)} \text { and } \eta_{i j}\left(\mathcal{U}^{(q)}\right)=\frac{\partial \omega_{j}^{(q)}}{\partial x_{i}}, \quad q=1,2
$$


Let us assume that $\Gamma_{u}, \Gamma_{0}, \Gamma_{c}$ and $\Gamma_{T}^{(q)}$ are pairwise nonintersecting smooth open subsurfaces of the boundary $\partial \Omega_{1} \cup \partial \Omega_{2} ;\left(\partial \Omega_{1} \cap \partial \Omega_{2}=\Gamma_{c}\right)$, while $\partial \Omega_{1}=$ $\bar{\Gamma}_{u} \cup \bar{\Gamma}_{T}^{(1)} \cup \Gamma_{c}, \partial \Omega_{2}=\bar{\Gamma}_{0} \cup \bar{\Gamma}_{T}^{(2)} \cup \Gamma_{c}$ and $\Gamma_{c}$ and $\Gamma_{u}$ have a positive measure. Let $(v)_{n}$ and $(v)_{t}$ denote the normal and the tangential component of the vector $v \in \mathbb{R}^{3}$, respectively. Below we will use the spaces $H^{s}\left(\Omega_{q}\right)$ and $H^{s}\left(\partial \Omega_{q}\right), s \in \mathbb{R}$, whose definitions and main properties can be found in [4], [5].

Definition 1. A vector function $\mathcal{U}^{(q)} \in\left(H^{1}\left(\Omega_{q}\right)\right)^{6}$ is called a weak solution of equation (3) for $\mathcal{F}^{(q)} \in\left(L_{2}\left(\Omega_{q}\right)\right)^{6}$ if $\mathcal{B}^{(q)}\left(\mathcal{U}^{(q)}, \Phi\right)=\left(\mathcal{F}^{(q)}, \Phi\right)_{0, \Omega_{q}}, \quad \forall \Phi \in$ $\left(C_{0}^{\infty}\left(\Omega_{q}\right)\right)^{6}$, where as usual

$$
(f, \varphi)_{0, \Omega}=\int_{\Omega} f \bar{\varphi} d x \quad(\bar{\varphi} \text { is complex conjugate to } \varphi) .
$$

Note that if $\mathcal{U}^{(q)} \in\left(H^{1}\left(\Omega_{q}\right)\right)^{6}$ and $\stackrel{(q)}{\mathcal{M}} \mathcal{U}^{(q)} \in\left(L_{2}\left(\Omega_{q}\right)\right)^{6}$, then $\left.\stackrel{(q)}{\mathcal{N}} \mathcal{U}^{(q)}\right|_{\partial \Omega_{q}}$ can be defined as an element of the space $\left(H^{-1 / 2}\left(\partial \Omega_{q}\right)\right)^{6}$ by using the relations:

$$
\left\langle\mathcal{N}^{(q)} \mathcal{U}^{(q)}, \Phi\right\rangle_{\partial \Omega_{q}}=\mathcal{B}^{(q)}\left(\mathcal{U}^{(q)}, \mathcal{V}\right)+\left(\stackrel{(q)}{\mathcal{M} \mathcal{U}^{(q)}}, \mathcal{V}\right)_{0, \Omega_{q}}, \quad \mathcal{V} \in\left(H^{1}\left(\Omega_{q}\right)\right)^{6},\left.\quad \mathcal{V}\right|_{\partial \Omega_{q}}=\Phi
$$

which take place for all $\Phi \in\left(H^{1 / 2}\left(\partial \Omega_{q}\right)\right)^{6}$; here and in what follows the symbol $\langle.,$.$\rangle denotes the duality relation between the corresponding dual spaces.$

Let us introduce the space

$$
\left(\widetilde{H}^{1 / 2}\left(\Gamma_{T}^{(q)}\right)\right)^{6}=\left\{\Phi \in\left(H^{1 / 2}\left(\partial \Omega_{q}\right)\right)^{6}:\left.\Phi\right|_{\partial \Omega_{q} \backslash \Gamma_{T}^{(q)}}=0\right\}
$$

and let $\left(H^{-1 / 2}\left(\Gamma_{T}^{(q)}\right)\right)^{6}$ be the dual space of this space. Now $\left.\stackrel{(q)}{\mathcal{N}} \mathcal{U}^{(q)}\right|_{\Gamma_{T}^{(q)}}$ can be defined as an element of the space $\left(H^{-1 / 2}\left(\Gamma_{T}^{(q)}\right)\right)^{6}$ by means of the formula

$$
\left\langle\left.\stackrel{\mathcal{N}}{\mathcal{U}}^{(q)}\right|_{\Gamma_{T}^{(q)}}, \Phi\right\rangle_{\Gamma_{T}^{(q)}}=\left\langle\stackrel{\mathcal{N}}{\mathcal{U}}^{(q)}, \Phi\right\rangle_{\partial \Omega_{q}}, \quad \forall \Phi \in\left(\widetilde{H}^{1 / 2}\left(\Gamma_{T}^{(q)}\right)\right)^{6}
$$

Similarly, we can define $\left.\left(\stackrel{(q)}{\sigma} \mathcal{U}^{(q)}\right)_{n^{(q)}}\right|_{\Gamma_{c}}$ as an element of the space $H^{-1 / 2}\left(\Gamma_{c}\right)$ :

$$
\begin{gathered}
\left\langle\left.\left(\stackrel{(q)}{\sigma} \mathcal{U}^{(q)}\right)_{n^{(q)}}\right|_{\Gamma_{c}},(v)_{n^{(q)}}\right\rangle_{\Gamma_{c}}=\mathcal{B}^{(q)}\left(\mathcal{U}^{(q)}, \mathcal{V}\right)+\left(\stackrel{\mathcal{M} \mathcal{U}^{(q)}}{ }, \mathcal{V}\right)_{0, \Omega_{q}}, \\
\mathcal{V}=(v, w) \in\left(H^{1}\left(\Omega_{q}\right)\right)^{6},\left.\quad w\right|_{\Gamma_{c}}=\left.(v)_{t^{(q)}}\right|_{\Gamma_{c}}=0,\left.\quad \mathcal{V}\right|_{\partial \Omega_{q} \backslash \Gamma_{c}}=0 .
\end{gathered}
$$

We will consider the folowing boundary-contact problem.

Problem 1. Find a vector function $\mathcal{U}^{(q)}=\left(u^{(q)}, w^{(q)}\right) \in\left(H^{1}\left(\Omega_{q}\right)\right)^{6}$ which is a weak solution of equation (3) and satisfies the conditions

$$
\begin{aligned}
& \left.\mathcal{U}^{(1)}\right|_{\Gamma_{u}}=0 \\
& \left.u^{(2)} \cdot n^{(2)}\right|_{\Gamma_{0}}=\left.\omega^{(2)} \cdot n^{(2)}\right|_{\Gamma_{0}}=0,\left.\quad\left(\stackrel{(2)}{\sigma} \mathcal{U}^{(2)}\right) t_{t^{(2)}}\right|_{\Gamma_{0}}=\left.\left(\stackrel{(2)}{\mu} \mathcal{U}^{(2)}\right)_{t^{(2)}}\right|_{\Gamma_{0}}=0
\end{aligned}
$$




$$
\begin{aligned}
& \left.\stackrel{(q)}{\mathcal{N}}\left(x, \partial, n^{(q)}\right) \mathcal{U}^{(q)}(x)\right|_{\Gamma_{T}^{(q)}}=\mathcal{P}^{(q)}(x) ; \\
& \left(u^{(1)} \cdot n^{(1)}+u^{(2)} \cdot n^{(2)}\right)_{\Gamma_{c}} \leq 0,\left.\quad\left(\stackrel{(2)}{\sigma} \mathcal{U}^{(2)}\right)_{n^{(2)}}\right|_{\Gamma_{c}}=\left.\left(\stackrel{(1)}{\sigma} \mathcal{U}^{(1)}\right)_{n^{(1)}}\right|_{\Gamma_{c}} \leq 0 ; \\
& \left\langle\left.\left(\stackrel{(1)}{\sigma} \mathcal{U}^{(1)}\right)_{n^{(1)}}\right|_{\Gamma_{c}}, \quad u^{(1)} \cdot n^{(1)}+u^{(2)} \cdot n^{(2)}\right\rangle_{\Gamma_{c}}=0 ; \\
& \left.\left(\stackrel{(1)}{\sigma} \mathcal{U}^{(1)}\right)_{t^{(1)}}\right|_{\Gamma_{c}}=\left.\left(\stackrel{(2)}{\sigma} \mathcal{U}^{(2)}\right)_{t^{(2)}}\right|_{\Gamma_{c}}=0 ; \\
& \left.\omega^{(1)}\right|_{\Gamma_{c}}=\left.\omega^{(2)}\right|_{\Gamma_{c}},\left.\stackrel{(1)}{\mu} \mathcal{U}^{(1)}\right|_{\Gamma_{c}}=-\left.\stackrel{(2)}{\mu} \mathcal{U}^{(2)}\right|_{\Gamma_{c}},
\end{aligned}
$$

where $n^{(2)}=-n^{(1)}$ on $\Gamma_{c}, \mathcal{F}^{(q)} \in\left(L_{2}\left(\Omega_{q}\right)\right)^{6}$ and $\mathcal{P}^{(q)} \in\left(H^{-1 / 2}\left(\partial \Omega_{q}\right)\right)^{6}$ such that $\left\langle\mathcal{P}^{(q)}, \Phi\right\rangle_{\partial \Omega_{q}}=0, \forall \Phi \in\left(H^{1 / 2}\left(\partial \Omega_{q}\right)\right)^{6},\left.\Phi\right|_{\bar{\Gamma}_{T}^{(q)}}=0$

A similar problem in the classical theory of elasticity is considered in [6].

Let us introduce the following notation:

$$
\begin{aligned}
& \mathcal{H}^{1}(\Omega)=\left\{\mathcal{U} \mid \mathcal{U}=\left(\mathcal{U}^{(1)}, \mathcal{U}^{(2)}\right) \in\left(H^{1}\left(\Omega_{1}\right)\right)^{6} \times\left(H^{1}\left(\Omega_{2}\right)\right)^{6}\right\} \\
& \|\mathcal{U}\|_{1, \Omega}^{2}=\sum_{q=1}^{2}\left\|\mathcal{U}^{(q)}\right\|_{1, \Omega_{q}}^{2} ; \mathcal{B}(\mathcal{U}, \mathcal{V})=\sum_{q=1}^{2} \mathcal{B}^{(q)}\left(\mathcal{U}^{(q)}, \mathcal{V}^{(q)}\right), \quad \forall \mathcal{U}, \mathcal{V} \in \mathcal{H}^{1}(\Omega) ; \\
& V=\left\{\mathcal{U} \in \mathcal{H}^{1}(\Omega)\left|\mathcal{U}^{(1)}\right|_{\Gamma_{u}}=0,\left.u^{(2)} \cdot n^{(2)}\right|_{\Gamma_{0}}=\left.\omega^{(2)} \cdot n^{(2)}\right|_{\Gamma_{0}}=0,\left.\quad \omega^{(1)}\right|_{\Gamma_{c}}=\left.\omega^{(2)}\right|_{\Gamma_{c}}\right\} ; \\
& \mathcal{K}=\left\{\mathcal{U} \in V \mid\left(u^{(1)} \cdot n^{(1)}+u^{(2)} \cdot n^{(2)}\right)_{\Gamma_{c}} \leq 0\right\} ; \\
& L(\mathcal{V})=(\mathcal{F}, \mathcal{V})_{0, \Omega}+\langle\mathcal{P}, \mathcal{V}\rangle_{\partial \Omega}=\sum_{q=1}^{2}\left[\left(\mathcal{F}^{(q)}, \mathcal{V}^{(q)}\right)_{0, \Omega_{q}}+\left\langle\mathcal{P}^{(q)}, \mathcal{V}^{(q)}\right\rangle_{\partial \Omega_{q}}\right] \\
& \mathcal{L}(\mathcal{V})=\frac{1}{2} \mathcal{B}(\mathcal{V}, \mathcal{V})-L(\mathcal{V}), \quad \forall \mathcal{V} \in \mathcal{H}^{1}(\Omega) .
\end{aligned}
$$

It is easy to prove that $\mathcal{U} \in \mathcal{K}$ minimizes the functional $\mathcal{L}$ on the convex closed set $\mathcal{K}$ if and only if

$$
\mathcal{B}(\mathcal{U}, \mathcal{V}-\mathcal{U}) \geq L(\mathcal{V}-\mathcal{U}), \quad \forall \mathcal{V} \in \mathcal{K}
$$

Definition 2. We call a vector function $\mathcal{U} \in \mathcal{K}$ a variational solution of Problem 1 if the variational inequality (8) is fulfilled.

Proposition 1. Each solution of Problem 1 is a variational one and vice versa.

Proof. Let $\mathcal{U} \in \mathcal{H}^{1}(\Omega)$ be a solution of Problem 1. By virtue of the internal regularization theorems (see [7]) we have $\mathcal{U} \in \mathcal{H}_{\text {loc }}^{2}(\Omega)$ and (3) holds. Now, applying the Green's formula,

$$
0=\sum_{q=1}^{2}\left[\left(\mathcal{M U}^{(q)}, \mathcal{V}^{(q)}\right)_{0, \Omega_{q}}+\left(\mathcal{F}^{(q)}, \mathcal{V}^{(q)}\right)_{0, \Omega_{q}}\right]=-\mathcal{B}(\mathcal{U}, \mathcal{V})+L(\mathcal{V})
$$




$$
+\left\langle\left.\stackrel{(2)}{\mathcal{N} \mathcal{U}^{(2)}}\right|_{\Gamma_{0}}, \mathcal{V}^{(2)}\right\rangle_{\Gamma_{0}}+\sum_{q=1}^{2}\left\langle\left.\stackrel{(q)}{\mathcal{N} \mathcal{U}^{(q)}}\right|_{\Gamma_{c}}, \mathcal{V}^{(q)}\right\rangle_{\Gamma_{c}}, \quad \forall \mathcal{V} \in V
$$

Since $\mathcal{U}$ is a solution of Problem 1 and $\mathcal{V} \in V$, the duality relation on $\Gamma_{0}$ is equal to zero, whereas the last term of the equality gives

$$
\begin{aligned}
\sum_{q=1}^{2}\left\langle\left.\stackrel{(q)}{\mathcal{N}} \mathcal{U}^{(q)}\right|_{\Gamma_{c}}, \mathcal{V}^{(q)}\right\rangle_{\Gamma_{c}}=\sum_{q=1}^{2}\left[\left\langle\left.\left(\stackrel{(q)}{\sigma} \mathcal{U}^{(q)}\right)_{n^{(q)}}\right|_{\Gamma_{c}}, v^{(q)} \cdot n^{(q)}\right\rangle_{\Gamma_{c}}\right. \\
\left.+\left\langle\left.\left(\stackrel{(q)}{\sigma} \mathcal{U}^{(q)}\right)_{t^{(q)}}\right|_{\Gamma_{c}},\left(v^{(q)}\right)_{t^{(q)}}\right\rangle_{\Gamma_{c}}+\left\langle\left.\stackrel{(q)}{\mu} \mathcal{U}^{(q)}\right|_{\Gamma_{c}}, w^{(q)}\right\rangle_{\Gamma_{c}}\right] \\
=\left\langle\left.\left(\stackrel{(1)}{\sigma} \mathcal{U}^{(1)}\right)_{n^{(1)}}\right|_{\Gamma_{c}}, v^{(1)} \cdot n^{(1)}+v^{(2)} \cdot n^{(2)}\right\rangle_{\Gamma_{c}}
\end{aligned}
$$

by virtue of the contact conditions of Problem 1 .

Thus eventually we obtain

$$
\mathcal{B}(\mathcal{U}, \mathcal{V})-L(\mathcal{V})=\left\langle\left.\left(\stackrel{(1)}{\sigma} \mathcal{U}^{(1)}\right)_{n^{(1)}}\right|_{\Gamma_{c}}, v^{(1)} \cdot n^{(1)}+v^{(2)} \cdot n^{(2)}\right\rangle_{\Gamma_{c}}, \quad \forall \mathcal{V} \in V
$$

Let $\mathcal{W} \in \mathcal{K}, \mathcal{W}=\left(\mathcal{W}^{(1)}, \mathcal{W}^{(2)}\right), \mathcal{W}^{(q)}=\left(\varphi^{(q)}, \psi^{(q)}\right)$. Then $\mathcal{V}=\mathcal{W}-\mathcal{U} \in V$ and, by virtue of conditions $(7)_{2}$, we have $\left(\stackrel{(1)}{\sigma} \mathcal{U}^{(1)}\right)_{n^{(1)}}=0$ at the points $\Gamma_{c}$ where $u^{(1)} \cdot n^{(1)}+u^{(2)} \cdot n^{(2)}<0$ whereas the inequality

$$
v^{(1)} \cdot n^{(1)}+v^{(2)} \cdot n^{(2)}=\varphi^{(1)} \cdot n^{(1)}+\varphi^{(2)} \cdot n^{(2)} \leq 0 \text { and }\left(\stackrel{(1)}{\sigma} \mathcal{U}^{(1)}\right)_{n^{(1)}} \leq 0
$$

is fulfilled if $u^{(1)} \cdot n^{(1)}+u^{(2)} n^{(2)}=0$.

Thus $\left\langle\left.\left(\stackrel{(1)}{\sigma} \mathcal{U}^{(1)}\right)_{n^{(1)}}\right|_{\Gamma_{c}}, v^{(1)} \cdot n^{(1)}+v^{(2)} \cdot n^{(2)}\right\rangle_{\Gamma_{c}} \geq 0$ and therefore $\forall \mathcal{W} \in \mathcal{K}:$ $\mathcal{B}(\mathcal{U}, \mathcal{W}-\mathcal{U})-L(\mathcal{W}-\mathcal{U}) \geq 0$.

Conversely, let now $\mathcal{U} \in \mathcal{K}$ be a solution of the variational inequaliy (8). After replacing in $(8) \mathcal{U}^{(q)} \pm \Phi^{(q)}$ by $\mathcal{V}^{(q)}$, where $\Phi^{(q)} \in\left(C_{0}^{\infty}\left(\Omega_{q}\right)\right)^{6}$, we obtain

$$
\mathcal{B}^{(q)}\left(\mathcal{U}^{(q)}, \Phi^{(q)}\right)=\left(\mathcal{F}^{(q)}, \Phi^{(q)}\right)_{0, \Omega_{q}}, \quad q=1,2,
$$

i.e., $\mathcal{U}^{(q)}$ is a weak solution of equation (3) and equation (3) is fulfilled again by virtue of the internal regularization theorems. Hence, applying the Green's formula and inequality (8), we get

$$
\begin{aligned}
\sum_{q=1}^{2}\left\langle\left.\stackrel{(q)}{\mathcal{N}} \mathcal{U}^{(q)}\right|_{\Gamma_{T}^{(q)}}\right. & \left.-\mathcal{P}^{(q)}, \mathcal{V}^{(q)}-\mathcal{U}^{(q)}\right\rangle_{\Gamma_{T}^{(q)}}+\sum_{q=1}^{2}\left\langle\left.\stackrel{(q)}{\mathcal{N} \mathcal{U}^{(q)}}\right|_{\Gamma_{c}}, \mathcal{V}^{(q)}-\mathcal{U}^{(q)}\right\rangle_{\Gamma_{c}} \\
& +\left\langle\left.\stackrel{(2)}{\mathcal{N}} \mathcal{U}^{(2)}\right|_{\Gamma_{0}}, \mathcal{V}^{(2)}-\mathcal{U}^{(2)}\right\rangle_{\Gamma_{0}} \geq 0
\end{aligned}
$$

On replacing in $(9) \mathcal{U}^{(q)} \pm \Phi^{(q)}$ by $\mathcal{V}^{(q)}$, where $\Phi^{(q)} \in\left(C^{\infty}\left(\Omega_{q}\right)\right)^{6}$ is such that $\left.\operatorname{supp} \Phi^{(q)}\right|_{\partial \Omega_{q}} \subset \Gamma_{T}^{(q)}$, we have $\langle\mathcal{N U}-\mathcal{P}, \Phi\rangle_{\Gamma_{T}^{(1)} \cup \Gamma_{T}^{(2)}}=0$.

This gives us (6). Condition (4) and the first conditions of (5), (7) 1 and $(7)_{4}$ are fulfilled automatically since $\mathcal{U} \in \mathcal{K}$. 
Let us now replace in $(9) \mathcal{V}$ by $\mathcal{U} \pm \Phi$, where $\Phi=\left(\Phi^{(1)}, \Phi^{(2)}\right) \in\left(C_{0}^{\infty}\left(\Omega_{1}\right)\right)^{6} \times$ $\left(C^{\infty}\left(\Omega_{2}\right)\right)^{6}$ is such that $\Phi^{(q)}=\left(\varphi^{(q)}, \psi^{(q)}\right),\left.\operatorname{supp} \Phi^{(2)}\right|_{\partial \Omega_{2}} \subset \Gamma_{0},\left.\psi^{(2)}\right|_{\Gamma_{0}}=0$ and $\left.\varphi^{(2)} \cdot n^{(2)}\right|_{\Gamma_{0}}=0$. Then $\left\langle\left.\left(\stackrel{(2)}{\sigma} \mathcal{U}^{(2)}\right)_{t^{(2)}}\right|_{\Gamma_{0}},\left(\varphi^{(2)}\right)_{t^{(2)}}\right\rangle_{\Gamma_{0}}=0$. Hence because of the arbitrariness of $\left(\varphi^{(2)}\right)_{t^{(2)}}$ it follows that $\left.\left(\stackrel{(2)}{\sigma} \mathcal{U}^{(2)}\right)_{t^{(2)}}\right|_{\Gamma_{0}}=0$.

In a similar manner we conclude that the condition $\left.\left(\stackrel{(2)}{\mu} \mathcal{U}^{(2)}\right)_{t^{(2)}}\right|_{\Gamma_{0}}=0$ is fulfilled on $\Gamma_{0}$.

The above reasoning enables us to rewrite (9) as

$$
\sum_{q=1}^{2}\left\langle\left.\left(\mathcal{N}^{(q)} \mathcal{U}^{(q)}\right)\right|_{\Gamma_{c}}, \mathcal{V}^{(q)}-\mathcal{U}^{(q)}\right\rangle_{\Gamma_{c}} \geq 0
$$

Choose $\mathcal{V}=\mathcal{U} \pm \Phi$ such that $\Phi=\left(\Phi^{(1)}, \Phi^{(2)}\right) \in \mathcal{H}^{1}(\Omega),\left.\operatorname{supp} \Phi^{(q)}\right|_{\partial \Omega_{q}} \subset$ $\bar{\Gamma}_{c}, \Phi^{(q)}=\left(\varphi^{(q)}, \psi^{(q)}\right),\left.\psi^{(1)}\right|_{\Gamma_{c}}=\left.\psi^{(2)}\right|_{\Gamma_{c}}=\left.\left(\varphi^{(1)}\right)_{t^{(1)}}\right|_{\Gamma_{c}}=\left.\left(\varphi^{(2)}\right)_{t^{(2)}}\right|_{\Gamma_{c}}=0$, and $\left.\varphi^{(1)} \cdot n^{(1)}\right|_{\Gamma_{c}}=-\left.\varphi^{(2)} \cdot n^{(2)}\right|_{\Gamma_{c}}=\psi$, where $\psi \in \widetilde{H}^{1 / 2}\left(\Gamma_{c}\right)$. Then

$$
\left\langle\left.\left(\stackrel{(1)}{\sigma} \mathcal{U}^{(1)}\right)_{n^{(1)}}\right|_{\Gamma_{c}}-\left.\left(\stackrel{(2)}{\sigma} \mathcal{U}^{(2)}\right)_{n^{(2)}}\right|_{\Gamma_{c}}, \psi\right\rangle_{\Gamma_{c}}=0, \quad \forall \psi \in \widetilde{H}^{1 / 2}\left(\Gamma_{c}\right),
$$

i. e., $\left.\left(\stackrel{(1)}{\sigma} \mathcal{U}^{(1)}\right)_{n}(1)\right|_{\Gamma_{c}}=\left.\left(\stackrel{(2)}{\sigma} \mathcal{U}^{(2)}\right)_{n^{(2)}}\right|_{\Gamma_{c}}$. In a similar way we establish condition $(7)_{3}$ and the second condition of $(7)_{4}$.

With the above arguments taken into account we can rewrite (10) as follows:

$$
\forall \mathcal{V} \in \mathcal{K}:\left\langle\left(\left.\left(\sigma^{(1)} \mathcal{U}^{(1)}\right)_{n^{(1)}}\right|_{\Gamma_{c}},\left(\left(v^{(1)}-u^{(1)}\right) \cdot n^{(1)}+\left(v^{(2)}-u^{(2)}\right) \cdot n^{(2)}\right)_{\Gamma_{c}}\right\rangle_{\Gamma_{c}} \geq 0 .\right.
$$

If we choose $\mathcal{U}=\mathcal{U}+\Phi$, where $\Phi=\left(\Phi^{(1)}, \Phi^{(2)}\right) \in \mathcal{H}^{1}(\Omega)$ is such that $\left.\operatorname{supp} \Phi^{(q)}\right|_{\partial \Omega_{q}} \subset \bar{\Gamma}_{c}, \Phi^{(q)}=\left(\varphi^{(q)}, \psi^{(q)}\right),\left.\psi^{(q)}\right|_{\Gamma_{c}}=0$ and $\left.\left(\psi^{(1)}\right)_{t^{(1)}}\right|_{\Gamma_{c}}=\left.\left(\varphi^{(2)}\right)_{t^{(2)}}\right|_{\Gamma_{c}}=$ $0,\left.\varphi^{(2)} \cdot n^{(2)}\right|_{\Gamma_{c}}=0$ and $\left.\varphi^{(1)} \cdot n^{(1)}\right|_{\Gamma_{c}}=\varphi$, where $\varphi \in \widetilde{H}^{1 / 2}\left(\Gamma_{c}\right)$ is arbitrary with the condition $\varphi \leq 0$, then $\mathcal{V} \in \mathcal{K}$ and $\left\langle\left.\left(\stackrel{(1)}{\sigma} \mathcal{U}^{(1)}\right)_{n^{(1)}}\right|_{\Gamma_{c}}, \varphi\right\rangle_{\Gamma_{c}}=0$. Hence $\left.\left({ }^{(1)} \mathcal{U}^{(1)}\right)_{n^{(1)}}\right|_{\Gamma_{c}} \leq 0$ as a distribution of the class $H^{-1 / 2}\left(\Gamma_{c}\right)$. Therefore the second condition of $(7)_{1}$ holds.

It remains to prove $(7)_{2}$.

Since $\mathcal{V}=0$ and $\mathcal{V}=2 \mathcal{U}$ belongs to the convex closed set $\mathcal{K}$ substituting $\mathcal{V}=0$ into $(11)$ and then doing the same with $\mathcal{V}=2 \mathcal{U}$, we obtain that $(7)_{2}$ is fulfilled everywhere on $\Gamma_{c}$. Proposition 1 is proved.

As is known, in the moment theory of elasticity the space of rigid displacement vectors is written as

$$
\mathcal{R}^{(q)}=\left\{\left(\rho^{(q)}, a^{(q)}\right) \mid \rho^{(q)}=\left[a^{(q)} \times x\right]+b^{(q)}, a^{(q)}, b^{(q)} \in \mathbb{R}^{3}, \quad q=1,2\right\},
$$

where $\left[a^{(q)} \times x\right]_{i}=\varepsilon_{i j k} a_{j}^{(q)} x_{k} ; \quad \mathcal{R}=\mathcal{R}^{(1)} \times \mathcal{R}^{(2)}$. 
It is easy to verify that $\mathcal{B}(\mathcal{U}, \mathcal{V})=0, \quad \forall \mathcal{U} \in \mathcal{R}$ and $\forall \mathcal{V} \in \mathcal{H}^{1}(\Omega)$.

The converse is also true: if $\mathcal{U} \in \mathcal{H}^{1}(\Omega)$ and $\xi_{i j}\left(\mathcal{U}^{(q)}\right)=0, \quad \eta_{i j}\left(\mathcal{U}^{(q)}\right)=$ $0, \quad i, j=1,2,3$, then $\mathcal{U} \in \mathcal{R}$.

Let $\mathcal{U} \in \mathcal{K}$ be a variational solution of Problem 1. Then if in (8) $\mathcal{V}$ is replaced by $\mathcal{U}+Y$, where $Y \in \mathcal{K} \cap \mathcal{R}$ (note that $\mathcal{U}+Y \in \mathcal{K}$ ), then we have $0=\mathcal{B}(\mathcal{U}, Y) \geq L(Y)$, i. e., the condition

$$
L(Y) \leq 0, \quad \forall Y \in \mathcal{K} \cap \mathcal{R},
$$

is the necessary one for the existence of a variational solution of Problem 1.

Lemma 1. Let $V \cap \mathcal{R}=\{0\}$ or $L(Y) \neq 0, \forall Y \in V \cap \mathcal{R} \backslash\{0\}$. Then there exists at most one variational solution of Problem 1.

Proof. Assume that $\mathcal{U}_{\mathcal{U}}$ and $\mathcal{\mathcal { U }}^{2}$ are solutions of inequality (8). Then

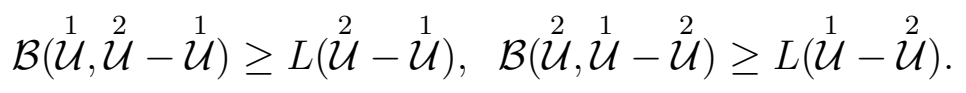

Hence $\mathcal{B}(\stackrel{1}{\mathcal{U}}-\stackrel{2}{\mathcal{U}}, \stackrel{1}{\mathcal{U}}-\stackrel{2}{\mathcal{U}}) \leq 0$.

This condition together with (2) implies that $\xi_{i j}(Y)=0, \quad \eta_{i j}(Y)=0, \quad Y=$ $\stackrel{1}{\mathcal{U}}-\stackrel{2}{\mathcal{U}}, \quad i, j=1,2,3$, i.e., $Y \in V \cap \mathcal{R}$. If by the assumption $V \cap \mathcal{R}=\{0\}$, then

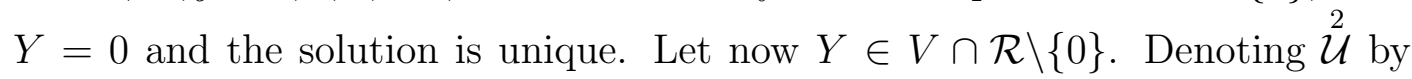
$\mathcal{U}$, we can write another arbitrary solution of (8) as $\mathcal{U}+Y$, where $Y \in V \cap \mathcal{R}$. Since the energy functional $\mathcal{L}$ has the same minimal value for the vectors $\mathcal{U}$ and $\mathcal{U}+Y$, we obtain

$$
[\mathcal{L}(\mathcal{U})=\mathcal{L}(\mathcal{U}+Y)] \Longrightarrow[L(\mathcal{U})=L(\mathcal{U}+Y)] \Longrightarrow L(Y)=0,
$$

which contradicts the condition of the lemma. Therefore $Y=0$ and the solution of (8) is unique.

As an example let us consider a plane contact problem, in which $\Gamma_{0}$ consists of segments parallel to the $x_{1}$-axis. In that case

$$
V \cap \mathcal{R}=\left\{Y=\left(Y^{(1)}, Y^{(2)}\right) \in \mathcal{H}^{1}(\Omega): Y^{(1)}=((0,0) ; 0), Y^{(2)}=\left(\left(b_{1}^{(2)}, 0\right) ; 0\right), b_{1}^{(2)} \in \mathbb{R}\right\}
$$

(it should be noted that in the two-dimensional case the space of rigid displacement vectors has the form

$$
\mathcal{R}=\left\{Y=\left(Y^{(1)}, Y^{(2)}\right) \in \mathcal{H}^{1}(\Omega): Y^{(q)}=\left(\left(b_{1}^{(q)}+a^{(q)} x_{2}, b_{2}^{(q)}-a^{(q)} x_{1}\right) ; a^{(q)}\right)\right\},
$$

where $a^{(q)} \in \mathbb{R}$ and $\left.b^{(q)}=\left(b_{1}^{(q)}, b_{2}^{(q)}\right) \in \mathbb{R}^{2}, q=1,2\right)$.

Let us assume that $n_{1}^{(2)} \geq 0$ throughout $\Gamma_{c}$ and $n_{1}^{(2)}(x)>0$ for some point $x \in \Gamma_{c}$. Then it is clear that $\mathcal{K} \cap \mathcal{R}=\left\{Y \in V \cap \mathcal{R}: b_{1}^{(2)} \leq 0\right\}$.

Condition (12), which is necessary for the existence of a variational solution, takes the form

$$
U_{1}^{(2)} \equiv \int_{\Omega_{2}} F_{1}^{(2)} d x+\left\langle\mathcal{P}_{1}^{(2)}, 1\right\rangle_{\Gamma_{T}^{(2)}} \geq 0 .
$$


By virtue of Lemma 1 , if $U_{1}^{(2)} \neq 0$, then there exists at most one solution of inequality (8) (indeed, $\forall Y \in V \cap \mathcal{R} \backslash\{0\}, L(Y)=b_{1}^{(2)} U_{1}^{(2)}, b_{1}^{(2)} \neq 0$, and if $U_{1}^{(2)} \neq 0$, then we have $\left.L(Y) \neq 0\right)$.

To obtain a general result of the existence of a variational solution of Problem 1 , we have to introduce the set of "bilateral" rigid displacement vectors

$$
\begin{array}{r}
\mathcal{R}^{*}=\left\{Y \in \mathcal{K} \cap \mathcal{R}: Y^{(1)}=(0 ; 0), Y^{(2)}=\left(b^{(2)} ; 0\right), b^{(2)} \in \mathbb{R}^{3}, 0 \in \mathbb{R}^{3},\right. \\
\left.\left.b^{(2)} \cdot n^{(2)}\right|_{\Gamma_{0}}=\left.b^{(2)} \cdot n^{(2)}\right|_{\Gamma_{c}}=0\right\} .
\end{array}
$$

The next theorem is an immediate corollary of Fichera's theorem on the existence of solutions of abstract variational inequalities (see [7], Theorem 1.II).

Theorem 1. Let $L(Y)) \leq 0, \quad \forall Y \in \mathcal{K} \cap \mathcal{R}$. If this condition is fulfilled in a strong sense, i.e., if the equality sign holds if and only if $Y \in \mathcal{R}^{*}$, then there exists a variational solution $\mathcal{U} \in \mathcal{K}$ of Problem 1 . Any other solution has the form $\mathcal{U}+Y$, where $Y \in V \cap \mathcal{R}$ is such that $\mathcal{U}+Y \in \mathcal{K}$ and $L(Y)=0$.

We will prove

Theorem 2. Let

$$
\begin{gathered}
V \cap \mathcal{R}=\mathcal{R}_{V}, \quad \mathcal{K} \cap \mathcal{R}=\mathcal{R}_{V} \\
L(Y)=0, \quad \forall Y \in \mathcal{R}_{V}
\end{gathered}
$$

and $V=\mathcal{R}_{V} \oplus H$.

Then there exists a unique solution $\widehat{\mathcal{U}} \in \widehat{\mathcal{K}}=\mathcal{K} \cap H$ of the problem

$$
\mathcal{L}(\widehat{\mathcal{U}}) \leq \mathcal{L}(\mathcal{Z}), \quad \forall \mathcal{Z} \in \widehat{\mathcal{K}}
$$

and each variational solution of Problem 1 can be represented as $\mathcal{U}=\widehat{\mathcal{U}}+$ $Y, \quad Y \in \mathcal{R}_{V}$.

If $\widehat{\mathcal{U}} \in \widehat{\mathcal{K}}$ is a solution of problem (15), then $\mathcal{U}=\widehat{\mathcal{U}}+Y$, where $Y$ is any element from $\mathcal{R}_{V}$, is a variational solution of Problem 1.

Proof. We begin by remarking that, in the case of a spatial problem, condition (13) is fulfilled if and only if $\Gamma_{0}$ is not a part of the plane (a part of the straight line in the two-dimensional case) or $\Gamma_{0}$ and $\Gamma_{c}$ are parallel plane subsulfaces (parallel segments). In the former case, $\mathcal{R}_{V}=\{0\}$ and condition (14) is fulfilled automatically, whereas in the latter case it holds if and only if $U_{1}^{(2)}=U_{2}^{(2)}=0$, where

$$
U_{j}^{(2)}=\int_{\Omega_{2}} F_{j}^{(2)} d x+\left\langle\mathcal{P}_{j}^{(2)}, 1\right\rangle_{\Gamma_{T}^{(2)}}, \quad j=1,2 .
$$

Let $H=V \ominus \mathcal{R}_{V}$, and the $P_{H}$ be the operator of orthogonal projection (in the sense of $\mathcal{H}^{1}$ ) of the space $V$ onto $H$. Using the second Korn inequality in the moment theory of elasticity (see [8]), we can prove that (see also [9])

$$
\forall \mathcal{V} \in H: \mathcal{B}(\mathcal{V}, \mathcal{V}) \geq c_{0}\left\|P_{H} \mathcal{V}\right\|_{1, \Omega}^{2}=c_{0}\|\mathcal{V}\|_{1, \Omega}^{2}
$$


where

$$
\|\mathcal{V}\|_{1, \Omega}^{2}=\sum_{q=1}^{2}\left\|\mathcal{V}^{(q)}\right\|_{1, \Omega_{q}}^{2}
$$

Hence we have

$$
\mathcal{L}(\mathcal{V})=\frac{1}{2} \mathcal{B}(\mathcal{V}, \mathcal{V})-L(\mathcal{V}) \geq \frac{c_{0}}{2}\|\mathcal{V}\|_{1, \Omega}^{2}-\|L\|\|\mathcal{V}\|_{1, \Omega}, \quad \forall \mathcal{V} \in H
$$

This proves the coercivity of the functional $\mathcal{L}$ on $H$.

Therefore problem (15) has a solution since $\mathcal{L}$ is a convex coercive functional on the convex closed set $\widehat{\mathcal{K}}=\mathcal{K} \cap H$. Let $\stackrel{1}{\mathcal{U}}$ and ${ }_{\mathcal{U}}^{2}$ be such two solutions. Then $\mathcal{Z}=\stackrel{1}{\mathcal{U}}-\stackrel{2}{\mathcal{U}} \in \mathcal{R}_{V} \cap H$, i. e., the solution is unique.

By (14) we have

$$
\mathcal{L}(\mathcal{V})=\mathcal{L}(\mathcal{V}+Y), \quad \forall Y \in \mathcal{R}_{V}
$$

Moreover, we can prove that

$$
P_{H}(\mathcal{K})=\widehat{\mathcal{K}} .
$$

Let now $\mathcal{U}$ be a variational solution of Problem (1). By (14)

$$
\mathcal{L}\left(P_{H} \mathcal{V}\right)=\mathcal{L}\left(P_{H} \mathcal{V}+P_{\mathcal{R}_{V}} \mathcal{V}\right) \mathcal{L}(\mathcal{V}), \quad \forall \mathcal{V} \in V
$$

Since by $(18) P_{H} \mathcal{U} \in \mathcal{K}$ and $\mathcal{L}\left(P_{H} \mathcal{U}\right)=\mathcal{L}(\mathcal{U}) \leq \mathcal{L}(\mathcal{V})=\mathcal{L}\left(P_{H} \mathcal{V}\right), \quad \forall \mathcal{V} \in \mathcal{K}$, we conclude with (18) taken into account that $P_{H} \mathcal{U}$ is a solution of problem (15).

Since we have already shown that problem (15) has a unique solution, we have $P_{H} \mathcal{U}=\widehat{\mathcal{U}}$ and the representation $\mathcal{U}=\widehat{\mathcal{U}}+Y, Y \in \mathcal{R}_{V}$ is obvious.

Conversely, let $\mathcal{U}=\widehat{\mathcal{U}}+Y$, where $\widehat{\mathcal{U}}$ is a solution of problem (15), and $Y \in \mathcal{R}_{V}$. Since $\mathcal{R}^{*}=\mathcal{R}_{V}$, we obtain

$$
\begin{aligned}
\left.\left(u^{(1)} \cdot n^{(1)}+u^{(2)} \cdot n^{(2)}\right)\right|_{\Gamma_{c}} & =\left.\left(\widehat{u}^{(1)} \cdot n^{(1)}+\widehat{u}^{(2)} \cdot n^{(2)}+y^{(1)} \cdot n^{(1)}+y^{(2)} \cdot n^{(2)}\right)\right|_{\Gamma_{c}} \\
& =\left.\left(\widehat{u}^{(1)} \cdot n^{(1)}+\widehat{u}^{(2)} \cdot n^{(2)}\right)\right|_{\Gamma_{c}} \leq 0
\end{aligned}
$$

and therefore $\mathcal{U} \in \mathcal{K}$.

By (17)

$$
\mathcal{L}(\mathcal{U})=\mathcal{L}(\widehat{\mathcal{U}}) \leq \mathcal{L}(\mathcal{Z}), \quad \forall \mathcal{Z} \in \widehat{\mathcal{K}}
$$

Clearly, $P_{H} \mathcal{V} \in \widehat{\mathcal{K}}, \forall \mathcal{V} \in \mathcal{K}$, and $\mathcal{L}(\mathcal{V})=\mathcal{L}\left(P_{H} \mathcal{V}+P_{\mathcal{R}_{V}} \mathcal{V}\right)=\mathcal{L}\left(P_{H} \mathcal{V}\right)$. Hence, by virtue of $(19)$ it follows that $\mathcal{L}(\mathcal{U}) \leq \mathcal{L}(\mathcal{V}), \quad \forall \mathcal{V} \in \mathcal{K}$, i. e., $\mathcal{U}$ is a variational solution of Problem 1.

The following theorem is true.

Theorem 3. Let

$$
\mathcal{R}^{*}=\{0\} \neq \mathcal{R}_{V}, \quad L(Y) \neq 0, \quad \forall Y \in \mathcal{R}_{V} \backslash\{0\},
$$

and either $\mathcal{K} \cap \mathcal{R}=\{0\}$ or

$$
\mathcal{L}(Y)<0, \quad \forall Y \in \mathcal{K} \cap \mathcal{R} \backslash\{0\} .
$$


Then $\mathcal{L}$ is coercive on $\mathcal{K}$ and there exists a unique variational solution of Problem 1.

Proof. Assume that $\mathcal{K} \cap \mathcal{R}=\{0\}$. Then the proof of the theorem immediately follows from the following lemma (see [10]).

Lemma 2. Let a semi-norm $|\cdot|$ be given on the Hilbert space $H$ with norm $\|\cdot\|_{H}$, and $\mathbb{R}=\{v \in H:|v|=0\}$ be a finite-dimensional subspace of $H$. It is assumed that for some $c_{i}>0, i=1,2$,

$$
c_{1}\|u\|_{H} \leq|u|+\left\|P_{\mathbb{R}} u\right\|_{H} \leq c_{2}\|u\|_{H}, \quad \forall u \in H,
$$

where $P_{\mathbb{R}}$ is the operator of orthogonal (in the sense of $H$ ) projection onto $\mathbb{R}$. $K$ is a convex closed subset of $H$ such that $K \cap \mathcal{R}=\{0\}$, and $\beta: H \rightarrow \mathbb{R}^{1}$ is the penalty functional for which $\operatorname{ker} \beta=K$ and

$$
D \beta(t u, v)=t D \beta(u, v), \quad \forall t>0, \quad \forall u, v \in H,
$$

where $D$ is the Gateaux differential.

Then

$$
|u|^{2}+\beta(u) \geq c\|u\|_{H}^{2}, \quad \forall u \in H .
$$

In our case, assuming that $H=V, \mathbb{R}=V \cap \mathcal{R}=\mathcal{R}_{V}$, we have

$$
|\mathcal{V}|=\sum_{q=1}^{2} \int_{\Omega_{q}}\left[\xi_{i j}\left(\mathcal{V}^{(q)}\right) \xi_{i j}\left(\mathcal{V}^{(q)}\right)+\eta_{i j}\left(\mathcal{V}^{(q)}\right) \eta_{i j}\left(\mathcal{V}^{(q)}\right)\right] d x
$$

and

$$
\beta(\mathcal{V})=\int_{\Gamma_{c}}\left[\left(v^{(1)} \cdot n^{(1)}+v^{(2)} \cdot n^{(2)}\right)^{+}\right]^{2} d s,
$$

where $\varphi^{+}=\min (\varphi, 0)$.

To check (22) we have to use the decomposition $V=\mathcal{R}_{V} \oplus Q$ and inequality (16). We have

$$
\begin{aligned}
\|\mathcal{V}\|_{1, \Omega}^{2} & =\left\|P_{Q} \mathcal{V}\right\|_{1, \Omega}^{2}+\left\|P_{\mathcal{R}_{V}} \mathcal{V}\right\|_{1, \Omega}^{2} \leq c\left|P_{Q} \mathcal{V}\right|^{2}+\left\|P_{\mathcal{R}_{V}} \mathcal{V}\right\|_{1, \Omega}^{2} \\
& =c|\mathcal{V}|^{2}+\left\|P_{\mathcal{R}_{V}} \mathcal{V}\right\|_{1, \Omega}^{2}, \quad \forall \mathcal{V} \in V,
\end{aligned}
$$

which gives the left-hand side of inequality (22). The right-hand side is obvious. It is also easy to check the fulfilment of $(23)$ and $\operatorname{ker} \beta=\mathcal{K}$. Now it is clear that (24) implies that $|\mathcal{V}|^{2} \geq c\|\mathcal{V}\|_{1, \Omega}^{2}, \quad \forall \mathcal{V} \in \mathcal{K}$. Hence the coercivity of the function $\mathcal{L}$ on $\mathcal{K}$ is obvious:

$$
\mathcal{L}(\mathcal{V})=\frac{1}{2} \mathcal{B}(\mathcal{V}, \mathcal{V})-L(\mathcal{V}) \geq \frac{c_{0}}{2}|\mathcal{V}|^{2}-L(\mathcal{V}) \geq c_{1}\|\mathcal{V}\|_{1, \Omega}^{2}-\|L\|\|\mathcal{V}\|_{1, \Omega}, \quad \forall \mathcal{V} \in \mathcal{K}
$$

Therefore Problem 1 has a variational solution.

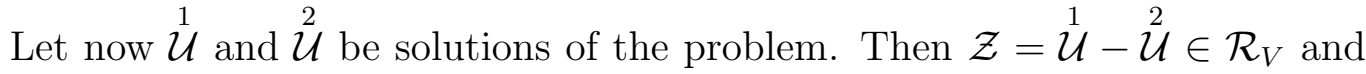

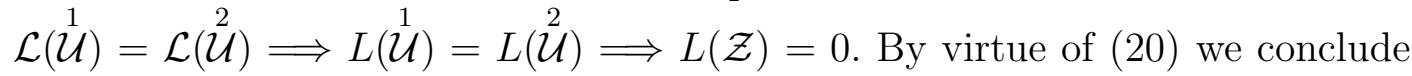
that $\mathcal{Z}=0$. 
Finally, if (21) is fulfilled, then the existence of a variational solution of Problem 1 is guaranteed by Theorem 1 (in our case $\mathcal{R}^{*}=\{0\}$ ). The uniqueness is proved similarly to the previous case.

Remark 1. If $V \cap \mathcal{R}=\{0\}$, then an equality of the Korn type $|\mathcal{V}| \geq c\|\mathcal{V}\|_{1, \Omega}$, $\forall \mathcal{V} \in V$, is valid and therefore $\mathcal{L}$ is coercive on the space $V$, which fact in its turn enables one to assert the existence and uniqueness of a variational solution of Problem 1.

\section{Problems with an Expanding Contact Zone}

We have considered the contact problem in which during deformation the contact zone does not extend beyond the boundary of some domain. However in some cases the contact zone may expand. Such a situation occurs when the domains $\Omega_{1}$ and $\Omega_{2}$ possess smooth boundaries in some neighborhood of the intersection $\partial \Omega_{1} \cap \partial \Omega_{2}$.

Let $Q \in \partial \Omega_{1} \cap \partial \Omega_{2}$ be an arbitrary point in the contact zone in the undeformed state. We introduce the local Cartesian system $(\xi, \eta)$ so that the $\xi$-axis be directed along $n^{(1)}$, and the $\eta$-axis along the tangent to the point $Q$. Let, furthermore, the parts of the boundary $\partial \Omega_{1}$ and $\partial \Omega_{2}$ which might come into contact during deformation be described in terms of local coordinates as $\Gamma_{c}^{(q)}=$ $\left\{(\xi, \eta) \mid \xi=f^{(q)}(\eta), \quad a \leq \eta \leq b\right\}, \quad q=1,2$, where $f^{(q)}$ are the continuous functions on $[a, b]$ (this segment is chosen so that it includes the projection of a possible contact zone onto the $\eta$-axis).

To avoid repetition, we do not formulate completely the contact problem which we are going to consider below (referred to as Problem 2) because it is formulated exactly like Problem 1 with the only difference that conditions $(7)_{1}$, $(7)_{2}$ and $(7)_{3}$ are replaced (see [6]) by the conditions

$$
\begin{array}{cc}
u_{\xi}^{(2)}-u_{\xi}^{(1)} \leq \varepsilon(\eta), \quad-\left(\stackrel{1)}{\sigma} \mathcal{U}^{(1)}\right)_{\xi}\left(\cos \alpha^{(1)}\right)^{-1}=\left(\stackrel{(2)}{\sigma} \mathcal{U}^{(2)}\right)_{\xi}\left(\cos \alpha^{(2)}\right)^{-1} \leq 0 ; & (7)_{1}^{\prime} \\
\left\langle\left(\stackrel{(2)}{\sigma} \mathcal{U}^{(2)}\right)_{\xi}, u_{\xi}^{(2)}-u_{\xi}^{(1)}-\varepsilon(\eta)\right\rangle=0 ; & (7)_{2}^{\prime} \\
\left(\stackrel{(1)}{\sigma} \mathcal{U}^{(1)}\right)_{\eta}=\left(\stackrel{(2)}{\sigma} \mathcal{U}^{(2)}\right)_{\eta}=0, & (7)_{3}^{\prime}
\end{array}
$$

where $\varepsilon(\eta)=f^{(1)}(\eta)-f^{(2)}(\eta), \eta \in[a, b]$, is a distance between the boundaries of the elastic bodies prior to deformations, $u_{\xi}^{(q)}$ are components of the dispacement vectors along the $\xi$-axis, $\alpha^{(q)}$ is the angle formed by the $\eta$-axis and the tangent to $\Gamma_{c}^{(q)}$, and $\cos \alpha^{(q)}=\left[1+\left(d f^{(q)} / d \eta\right)^{2}\right]^{-1 / 2}$.

Let us again introduce the space $V$ and the convex closed set $\mathcal{K}_{\varepsilon}$ as follows:

$$
\begin{aligned}
& V=\left\{\mathcal{V} \in \mathcal{H}^{1}(\Omega) ;\left.\mathcal{V}^{(1)}\right|_{\Gamma_{u}}=0,\left.v^{(2)} \cdot n^{(2)}\right|_{\Gamma_{0}}=\left.w^{(2)} \cdot n^{(2)}\right|_{\Gamma_{0}}=0,\right. \\
&\left.\left.w^{(1)}\right|_{\Gamma_{c}^{(1)}}=\left.w^{(2)}\right|_{\Gamma_{c}^{(2)}} \text { with the same coordinate } \eta \in[a, b]\right\}, \\
& \mathcal{K}_{\varepsilon}=\left\{\mathcal{V} \in V ; v_{\xi}^{(2)}-v_{\xi}^{(1)} \leq \varepsilon(\eta), \eta \in[a, b]\right\} .
\end{aligned}
$$

The functionals $L$ and $\mathcal{L}$ are the same as before. 
Definition 3. A function $\mathcal{U} \in \mathcal{K}_{\varepsilon}$ is called a variational solution of Problem 2 if $\mathcal{L}(\mathcal{U}) \leq \mathcal{L}(\mathcal{V}), \quad \forall \mathcal{V} \in \mathcal{K}_{\varepsilon}$

Our next proposition below is proved quite similarly to Proposition 1.

Proposition 2. Each solution of Problem 2 is a variational solution of this problem and vice versa.

Let formulate without proving a result similar to Theorem 2.

Theorem 4. Let

$$
\begin{aligned}
& \mathcal{K}_{0}=\left\{\mathcal{V} \in V ; v_{\xi}^{(2)}-v_{\xi}^{(1)} \leq 0, \forall \eta \in[a ; b]\right\} \\
& L(Y)=0, \quad \forall Y \in \mathcal{R}_{V} \equiv \mathcal{K}_{0} \cap \mathcal{R} .
\end{aligned}
$$

and $V=H \oplus \mathcal{R}_{V}$ be the orthogonal decomposition of the space $V$.

Then $\mathcal{L}$ is coercive on $H$ and there exists a unique solution $\widehat{\mathcal{U}} \in \widehat{\mathcal{K}}_{\varepsilon}=\mathcal{K}_{\varepsilon} \cap H$ of the problem

$$
\mathcal{L}(\widehat{\mathcal{U}}) \leq \mathcal{L}(\mathcal{Z}), \quad \forall \mathcal{Z} \in \widehat{\mathcal{K}}_{\varepsilon}
$$

Moreover, each variational solution of Problem 2 can be written in the form $\mathcal{U}=\widehat{\mathcal{U}}+Y$, where $\widehat{\mathcal{U}}$ is a solution of $(25)$ and $Y \in \mathcal{R}_{V}$. If $\widehat{\mathcal{U}} \in \mathcal{R}_{V}$ is a solution of (25), then $\widehat{\mathcal{U}}+Y$ is a variational solution of Problem 2 for any $Y \in \mathcal{R}_{V}$.

The proof of this theorem repeats that of Theorem 2. Finally, the following theorem is valid.

Theorem 5. Let $\Gamma_{0}$ consist of segments parallel to the $x_{1}$-axis, $\cos \left(\xi, x_{1}\right)>0$ and

$$
U_{1}^{(2)}=\int_{\Omega_{2}} F_{1}^{(2)} d x+\left\langle\mathcal{P}_{1}^{(2)}, 1\right\rangle>0 .
$$

Then $\mathcal{L}$ is coercive on $\mathcal{K}_{\varepsilon}$ and there exists a unique solution of Problem 2.

Proof. Let

$$
Q(\mathcal{V})=\int_{a}^{b}\left(v_{\xi}^{(2)}-v_{\xi}^{(1)}\right) d \eta \text { and } V_{Q}=\{\mathcal{V} \in V ; Q(\mathcal{V})=0\}
$$

Then

$$
\mathcal{R} \cap V_{Q}=\{0\}
$$

Indeed,

$$
\mathcal{R} \cap V_{Q} \subset \mathcal{R}_{V}=\left\{Y=\left(Y^{(1)}, Y^{(2)}\right) ; Y^{(2)}=((0,0) ; 0), Y^{(2)}=\left(\left(b_{1}, 0\right) ; 0\right), b_{1} \in \mathcal{R}^{1}\right\} .
$$

Since $Q(Y)=0$, we have

$$
0=b_{1} \int_{a}^{b} \cos \left(\xi, x_{1}\right) d \eta \Longrightarrow b_{1}=0 .
$$


Using (27) and the second Korn inequality, one can prove that

$$
|\mathcal{V}| \geq c\|\mathcal{V}\|_{1, \Omega}, \quad \forall \mathcal{V} \in V_{Q}
$$

Let $\mathcal{V} \in V$. Choosing $Y \in \mathcal{R}_{V}$ such that $Y^{(1)}=((0,0) ; 0), \quad Y^{(2)}=$ $\left(\left(Q(\mathcal{V}) d^{-1}, 0\right) ; 0\right)$, where

$$
d=\int_{a}^{b} \cos \left(\xi, x_{1}\right) d \eta
$$

observing that the equality

$$
Q(P \mathcal{V})=Q(\mathcal{V})-Q(Y)=Q(\mathcal{V})-\int_{a}^{b} Q(\mathcal{V}) d^{-1} \cos \left(\xi, x_{1}\right) d \eta=0
$$

holds for the difference $P \mathcal{V}=\mathcal{V}-Y$, we find that $P \mathcal{V} \in V_{Q}$. By (28) we have

$$
\begin{aligned}
\mathcal{L}(\mathcal{V}) & =\frac{1}{2} \mathcal{B}(P \mathcal{V}, P \mathcal{V})-L(P \mathcal{V})-L(Y) \\
& \geq c_{1}\|P \mathcal{V}\|_{1, \Omega}^{2}-c_{2}\|P \mathcal{V}\|_{1, \Omega}-Q(\mathcal{V}) d^{-1} U_{1}^{(2)} .
\end{aligned}
$$

Clearly, if $\|\mathcal{V}\|_{1, \Omega} \longrightarrow \infty$, then at least one of the norms $\|P \mathcal{V}\|_{1, \Omega}$ or $\|Y\|_{1, \Omega}$ tends to infinity. Moreover,

$$
\begin{aligned}
& \mathcal{V} \in \mathcal{K}_{\varepsilon} \Longrightarrow Q(\mathcal{V}) \leq \int_{a}^{b} \varepsilon(\eta) d \eta<+\infty \\
& \|Y\|_{1, \Omega}=|Q(\mathcal{V})| d^{-1}\left(\int_{\Omega_{2}} d x\right)^{1 / 2}=|Q(\mathcal{V})| d^{-1}\left(\operatorname{mes} \Omega_{2}\right)^{1 / 2}
\end{aligned}
$$

1. Let $\|Y\|_{1, \Omega} \longrightarrow \infty$. From (30) and (31) it follows that $-Q(\mathcal{V}) \longrightarrow+\infty$.

Since $c_{1}\|P \mathcal{V}\|_{1, \Omega}^{2}-c_{2}\|P \mathcal{V}\|_{1, \Omega} \geq c_{3}>-\infty,(29)$ and (26) imply that $\mathcal{L}(\mathcal{V}) \longrightarrow$ $+\infty$.

2. Let now $\|P \mathcal{V}\|_{1, \Omega} \longrightarrow \infty$. Then (30) yields

$$
c_{1}\|P \mathcal{V}\|_{1, \Omega}^{2}-c_{2}\|P \mathcal{V}\|_{1, \Omega} \longrightarrow+\infty, \quad-Q(\mathcal{V}) d^{-1} U_{1}^{(2)} \geq-d^{-1} U_{1}^{(2)} \int_{a}^{b} \varepsilon d \eta>-\infty .
$$

Hence, by (29), we obtain $\mathcal{L}(\mathcal{V}) \longrightarrow+\infty$. Thus we have proved the coercivity of the functional $\mathcal{L}$ on $\mathcal{K}_{\varepsilon}$, which in its turn guarantees the existence of a solution of Problem 2. Let $\stackrel{1}{\mathcal{U}}$ and $\stackrel{2}{\mathcal{U}}$ be any two solutions. Then $\mathcal{Z}=\stackrel{1}{\mathcal{U}}-\stackrel{2}{\mathcal{U}} \in \mathcal{R}_{V}$ and $L(\mathcal{Z})=0$. On the other hand, $L(\mathcal{Z})=b U_{1}^{(2)}, b \in \mathcal{R}^{1}$, and (26) implies that $b=0$, i. e., $\mathcal{Z}=0$. The uniqueness and, accordingly, Theorem 5 is proved. 


\section{REFERENCES}

1. I. HlavaČEK and M. HlavaČEK, Om the existence and uniqueness of solution and some variational principles in linear theories of elasticity with couple-stress. Aplikace Matematiky 14(1969), 387-410.

2. V. D. Kupradze, T. G. Gegelia, M. O. Basheleishvili, and T. V. Burchuladze, Three-dimensional problems of the mathematical theory of elasticity and thermoelasticity. North-Holland Series in Applied Mathematics and Mechanics, v. 25, North-Holland Publ. Co., Amsterdam - New York - Oxford, 1979; Russian original: Nauka, Moscow, 1976.

3. O. I. Maisaya and R. I. Gachehiladze, Investigation of noncorrect problems of the classical and the moment theory of elasticity. (Russian) Trudy Tbiliss. Mat. Inst. Razmadze 87(1987), 114-125.

4. J.-L. Lions, E. Magenes, Problemes aux limites non homogenes et applications. Vol. 1, 2. Dunod, Paris, 1968; Russian transl.: Mir, Moscow, 1971.

5. G. Duvant and J.-L. Lions, les inéquations en mécanique et en physique. Dunod, Paris, 1972; Russian transl.: Nauka, Moscow, 1980.

6. I. Hlavaček, J. Haslinger, J. NecǍs, and J. Lovišek, Solution of variational inequalities in mechanics. (Slovak) ALFA, Bratislava, 1982; Russian transl,: Mir, Moscow, 1986.

7. G. FicherA, Existence theorems in elasticity. Hand. d. Physik, Bd. 1/2, No. 3, SpringerVerlag, Heidelberg, 1972; Russian transl.: Mir, Moscow, 1974.

8. O. I. Maisaia, Existence theorems in the moment theory of elasticity. (Russian) Soobshch. Akad. Nauk Gruz. SSR 75(1974), No. 2, 293-296.

9. I. HlavaČEK and J. NeČAs, On inequalities of Korn's type, I, II. Arch. Ration. Mech. Anal. 36(1970), 305-334.

10. J. NEČAs, On regularity of solutions to nonlinear variational inequalities for second-order elliptic systems. Collection of articles dedicated to Mauro Picone on the occasion of his ninetieth birthday, II. Rend. Mat. (6) 8(1975), No. 2, 481-498.

(Received 25.08.2000; revised 14.08.2001)

Author's address:

A. Razmadze Mathematical Institute

Georgian Academy of Sciences

1, Aleksidze St., Tbilisi 380093

Georgia 